\title{
Andrei Okounkov to Deliver a Public Lecture at 8ECM
}

Tomaž Pisanski (University of Primorska, Koper, Slovenia)

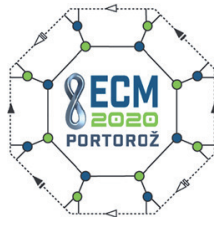

$8^{\text {th }}$ EUROPEAN CONGRESS QF MATHEMATICS 2020 D. 11 July 2020

We are happy to announce that Fields medalist Andrei Okounkov will deliver a public lecture at the 8th European Congress of Mathematics!

Andrei Okounkov is a Russian mathematician who works in mathematical physics and neighbouring areas of representation theory and algebraic geometry. Enumerative geometry lies at the crossroads of all of these fields of mathematics, and a lot of Okounkov's recent work focuses on K-theoretic generalisations of classical questions in enumerative geometry. In particular, a K-theoretic generalisation of the Donaldson-Thomasstyle counting of curves in algebraic threefolds is an exciting area at the forefront of current research with a conjectural relation to counting membranes of M-theory put forward by Nekrasov and Okounkov, and a geometric representation theory description of its basic building blocks obtained by Okounkov and A.Smirnov. Earlier conjectures of Maulik-Nekrasov-Okounkov-Pandharipande, connecting cohomological DT counts with Gromov-Witten theory of algebraic threefolds, in many ways shaped the developments in both fields. The proof of the MNOP conjectures for toric varieties by MaulikOblomkov-Okounkov-Pandharipande and the work that followed extends, among other things, the representation theoretic understanding of the Gromov-Witten theory of curves (and also of the point) obtained in the early 2000s by Okounkov and Pandharipande.

In 2004, Okounkov was awarded an EMS prize for work that "contributed greatly to the field of asymptotic combinatorics." In 2006, at the 25th International Congress of Mathematicians in Madrid, Spain, he received the Fields medal "for his contributions to bridging probability, representation theory and algebraic geometry."

Andrei Okounkov is a professor at the Columbia University in the city of New York and at the Skolkovo Institute of Science and Technology in Moscow. He also serves as the academic supervisor of HSE International Laboratory of Representation Theory and Mathematical Physics. His previous positions include the University of Chicago, University of California at Berkeley, and Princeton University.

We look forward to welcoming Professor Okounkov at the 8th European Congress of Mathematics in Portorož, Slovenia!

Further details will be posted on our website, please follow us on $8 \mathrm{ecm}$.si for more news.

The photo and CV of Tomaž Pisanski can be found in previous Newsletter issues. 